\title{
Improving attendance for cardiovascular risk assessment in Australian general practice: an RCT of a monetary incentive for patients
}

\author{
Nigel Stocks ${ }^{1 *}$, James Allan², Oliver Frank1, Sue Williams ${ }^{3}$ and Philip Ryan ${ }^{3}$
}

\begin{abstract}
Background: Preventive health care is an important part of general practice however uptake of activities by patients is variable. Monetary incentives for doctors have been used in the UK and Australia to improve rates of screening and immunisation. Few studies have focussed on incentives for patients to attend preventive health care examinations. Our objective was to investigate the use of a monetary incentive to increase patient attendance with their general practitioner for a cardiovascular risk assessment (CVRA).
\end{abstract}

Methods: A pragmatic RCT was conducted in two Australian general practices. Participating GPs underwent academic detailing for cardiovascular risk assessment. 301 patients aged 40-74, who did not have cardiovascular disease, were independently randomised to receive a letter inviting them to a no cost cardiovascular risk assessment with their GP, or the same letter plus an offer of a $\$ 25$ shopping voucher if they attended. An audit of patient medical records was also undertaken and a patient questionnaire administered to a sub sample of participants. Our main outcome measure was attendance for cardiovascular risk assessment.

Results: In the RCT, 56/301(18.6\%) patients attended for cardiovascular risk assessment, 29/182 (15.9\%) in the control group and 27/119 (22.7\%) in the intervention group. The estimated difference of $6.8 \%$ (95\% Cl: $-2.5 \%$ to 16.0\%) was not statistically significant, $P=0.15$. The audit showed that GPs may underestimate patients' absolute cardiovascular risk and the questionnaire that mailed invitations from GPs for a CVRA may encourage patients to attend.

Conclusions: A small monetary incentive does not improve attendance for cardiovascular risk assessment. Further research should be undertaken to determine if there are other incentives that may increase attendance for preventive activities in the general practice setting.

Clinical trials registration: ACTRN12608000183381

\section{Backgroud}

Most Primary Health Care (PHC) interventions to improve the primary and secondary prevention of cardiovascular disease (CVD) have focussed on improvements in identifying patients at risk [1] and multi-factorial interventions, usually incorporating nurse led clinics for secondary prevention [2]. Whilst GPs in Australia and the UK have been offered financial incentives to reach quality improvement targets, little research has explored what incentives might encourage consumers to attend GPs for preventive

\footnotetext{
*Correspondence: nigel.stocks@adelaide.edu.au

'Discipline of General Practice, The University of Adelaide, Adelaide, SA 5005, Australia

Full list of author information is available at the end of the article
}

health care. Some research has examined incentives to improve preventive behaviour, for instance to increase rates of immunisation, cancer screening and smoking cessation $[3,4]$. Only one general practice study conducted in Denmark showed that attendance at preventive health examinations for CVD was higher when the examination was free [5]. There have been no Australian or UK studies exploring the use of incentives to promote preventive health examinations.

Our study aimed to trial the use of a monetary incentive to increase the uptake of a cardiovascular risk assessment (CVRA) in patients without a history of CVD.

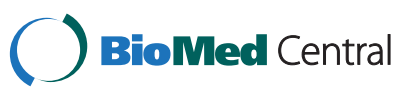




\section{Methods}

\section{Study type and setting}

A randomised controlled trial of a monetary incentive to improve attendance for CVRA was conducted in two Adelaide urban general practices (A and B). Both practices had similar demographic and socioeconomic characteristics (Index of socio-economic advantage-disadvantage for the top 10 postcodes of patients in each practice 984 and 985 respectively).

\section{Participants}

Practice databases were searched to identify patients aged 40-74 years without diagnosed CVD. GPs reviewed the generated patient lists to exclude those that they considered unsuitable because of serious illness, language barriers, or recent bereavement that might affect their participation in the study. The list was further limited to include only one patient per household, by alternately selecting a male or female patient from a shared address.

\section{Intervention}

Letters were sent to a total of 301 patients inviting them to attend for CVRA with their usual GP. Intervention arm patients $(n=119)$ were offered a shopping voucher (to the value of \$25) for attendance within 6 months of the invitation date. Along with the invitation, all patients received an information sheet, consent form and a brochure outlining what a CVRA entailed. Attendance slips were also provided, to be taken to the assessment visit to identify participants as part of the trial, and to act as evidence of attendance. These slips were collected during regular visits to the practices. Patients had 6 months in which to attend for CVRA after the invitation was sent. Reminders were not sent. All GPs were trained in the use of a paper version of the NZ absolute cardiovascular risk tables [6].

\section{Outcome}

Attendance at the general practice for a CVRA within 6 months of the invitation, as verified by collection of an attendance slip by research staff during a 9 month follow up period.

\section{Randomisation}

Within each practice eligible patients were randomised to control or intervention arms using a random number table by an independent statistician. Assignment was weighted to the control arm (3:2) because a lower participation rate was expected in this arm.

\section{Sample size calculation}

We assumed that $65 \%$ of patients would respond to a letter asking them to attend for CVRA without any incentive. To be able to detect an increase in the response rate above $80 \%$ (a $15 \%+$ absolute increase) in those receiving an incentive, with 95\% confidence and $80 \%$ power we calculated that we would need 150 patients from each practice (300 in total).

\section{Audit and questionnaire}

An audit of consenting participants' medical records was conducted between 3 and 9 months after their attendance for the CV risk assessment. Details collected included attendance date, gender, age, family history of CVD, diabetes, smoking status, height, weight, blood pressure, lipid analysis, current medications and relevant referrals. The audit also recorded any CV risk calculation made at the risk assessment visit, or changes to medications at, or since, the visit. Dates of the most recent previous consultation, and any subsequent consultations were also recorded.

A follow-up questionnaire was sent to a subgroup of participants (practice B) to ask why they participated in the trial (not reported here), provide an estimate of their own CVD risk, the perceived benefit of CVRA and whether they would have seen their GP for a CVRA 'heart health check' if they had not been invited.

\section{Analysis}

Descriptive statistics were used to report most data. A binomial generalised linear model with an identity link was used to estimate the difference in the proportions of patients attending between the intervention and control groups. The standard error of the estimate was not adjusted for clustering at the practice level, as we had only two practices and no suitable method exists to adjust in these circumstances. It is possible therefore that our test may be anti-conservative.

\section{Ethics}

This study received ethics approval from the University of Adelaide Human Research Ethics Committee. Informed consent was obtained from all participants and patients only entered into the trial if we received a signed consent form by a reply paid envelop. Some patients consented to be in the trial but did not consent to the medical record audit.

\section{Results}

Attendance frequencies, as determined from collection of the attendance slips, are shown in Table 1 . Fifty nine percent of all participants were female; and 57\% were aged over 60 years. A CONSORT flow chart appears in Figure 1.

The estimated absolute difference of $6.8 \%$ (95\% CI: $-2.5 \%$ to $16.0 \%$ ) was not statistically significant, $\mathrm{P}=0.15$ using a binominal generalised linear model. 
Table 1 Attendance for cardiovascular risk assessment

\begin{tabular}{ll}
\hline Total no Attendees/Invited & Combined \\
\hline Control Arm Attended/Invited & $56 / 301(18.6 \%)$ \\
\hline Intervention Arm attended/Invited & $29 / 182(15.9 \%)$ \\
\hline
\end{tabular}

There were no adverse events or side effects in either the intervention or control group.

\section{Characteristics of attendees}

The age and gender of participants attending for CVRA is summarised in Table 2. The data presented indicates a similar age and gender distribution by practice and intervention group, although there were slightly fewer control group participants in the $40-49$ age band and slightly more in the 70-74 age group.

\section{Audit}

The audit of 41 (consenting) participants medical records (out of 56 participants) enabled us to determine the time between their most recent previous consultation and their attendance for CVRA, as an indicator of their frequency of attendance. Most of these participants (68\%) had attended the practice within 60 days prior to their attendance for CVRA (Figure 2), suggesting that the majority of our participants were regularly attending patients. Confirmation of attendance dates also indicated that $85 \%$ of participants attended within 2 months of invitation and all within 6 months. Time to attendance was similar for control and intervention groups.

\section{Comparison of GP and audit assessed risk}

Using data collected by an audit of the GP records of 41 participants we compared the GPs' risk score recorded

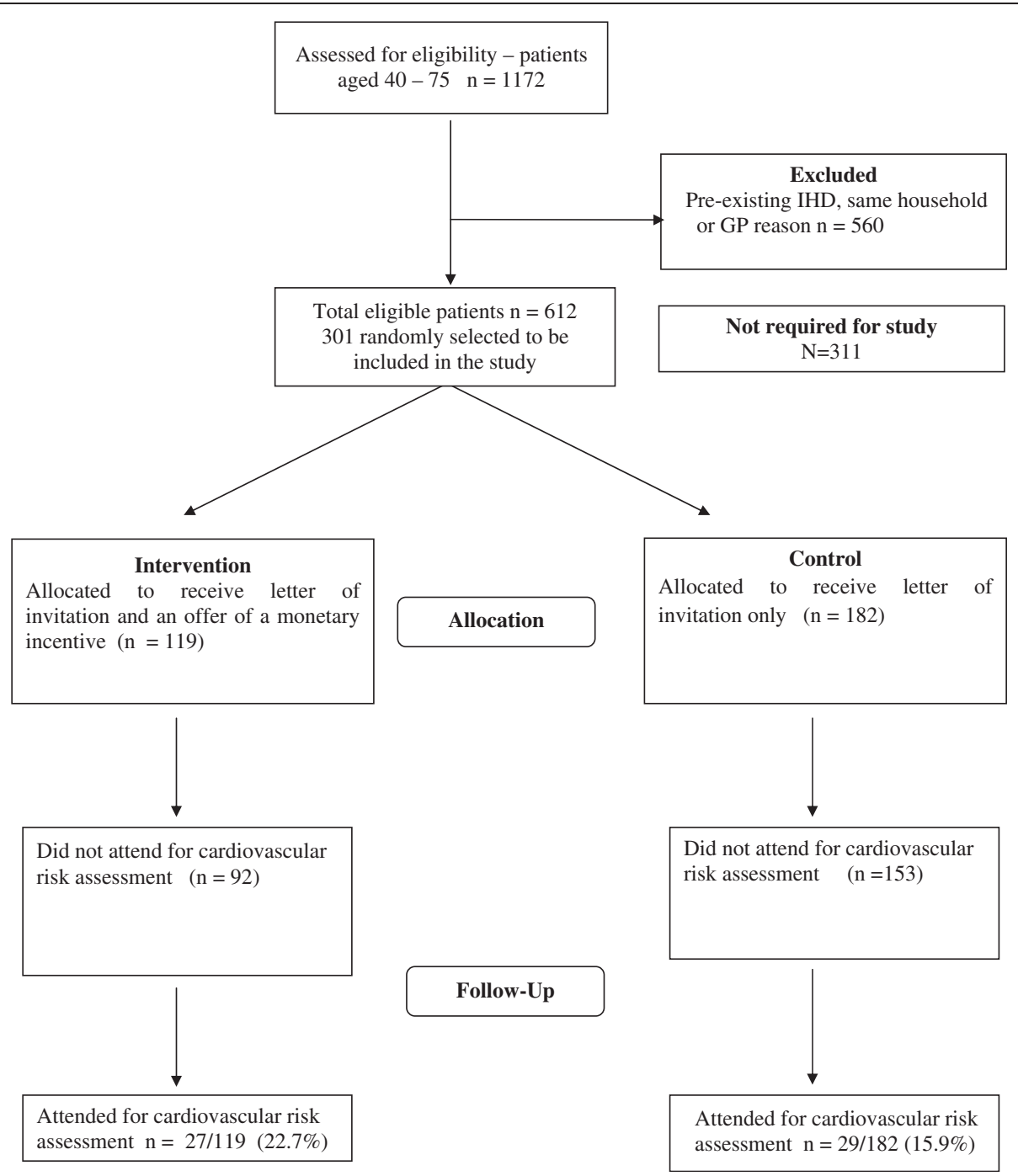

Figure 1 Study flow chart. 
Table 2 Characteristics of attendees for CVD risk assessment

\begin{tabular}{llll}
\hline & Practice A (I:C) & Practice B (I:C) & Combined (I:C) \\
\hline Gender & & & \\
\hline Female & $14(6: 8) 58 \%$ & $19(10: 9) 59 \%$ & $33(16: 17) 59 \%$ \\
\hline Male & $10(6: 4) 42 \%$ & $13(5: 8) 41 \%$ & $23(11: 12) 41 \%$ \\
\hline Totals & $24100 \%$ & $32100 \%$ & $56100 \%$ \\
\hline Age (years) & & & \\
\hline $40-49$ & $5(4: 1) 21 \%$ & $5(3: 2) 16 \%$ & $10(7: 3) 18 \%$ \\
\hline $50-59$ & $7(3: 4) 29 \%$ & $7(4: 3) 21 \%$ & $14(7: 7) 25 \%$ \\
\hline $60-69$ & $7(4: 3) 29 \%$ & $9(3: 6) 27 \%$ & $16(7: 9) 29 \%$ \\
\hline $70-74$ & $5(1: 4) 21 \%$ & $11(5: 6) 33 \%$ & $16(6: 10) 29 \%$ \\
\hline Totals & $24100 \%$ & $32100 \%$ & $56100 \%$ \\
\hline
\end{tabular}

during the CVRA visit (available for only 34 attendees) with one that we calculated from the available data in the medical records. Figure 3 shows a trend toward lower absolute CVD risk estimates by the GPs.

\section{Questionnaire}

Questionnaires were sent to 32 participants from practice B and we received 24 responses (75\%) Participants were asked to indicate their level of risk (from extremely low risk to extremely high risk) by marking a cross on a visual analogue scale. Their responses were compared, for intervention (I) and control (C) groups and summarised in Table 3 below. They were also asked if they would have had the CVD check up for their 'heart health' if they had not received an invitation to visit their GP, 12 of 23 respondents said no.

\section{Discussion}

\section{Main results}

The results of our RCT showed that attendance after a mailed invitation to patients aged 40-75 for CVRA was low (15.9\%) and that a $\$ 25$ monetary incentive did not lead to a statistically significantly increase in attendance, although there was an absolute difference in attendance between the two groups of $6.8 \%$. We also found that

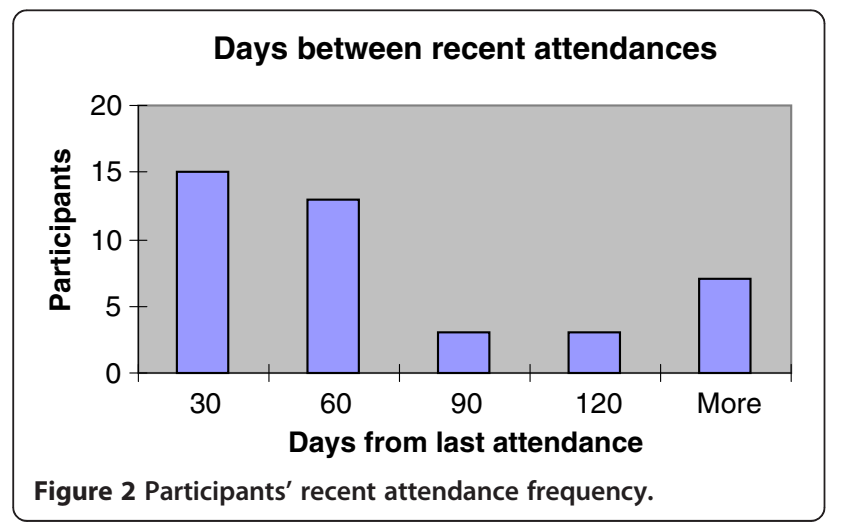

GPs in this study tended to underestimate their patients CVD risk based on available information in the medical records, that participants in the intervention and control groups had similar perceptions of their future CVD risk and that almost half the participants from one practice would not have had a CVRA without the prompt provided by the invitation letter.

Our desired behaviour was attendance for CVRA, which has been promoted recently [7]. Although the cost-effectiveness of CVRA is yet to be determined CVRA can facilitate the management of individual patients [8]. We could have chosen the 45-49 year old health check, a separate item number in the Australian fee for service system (\$104.00 AUS), or any other preventive health activity, but the CVRA was applicable to everyone in the selected age range, except those with established CVD. We were interested in a proof of principle and although responses may vary between desired activities the addition of an appropriate incentive might be expected to improve response rates.

We can only speculate why the uptake for CVRA was low and why a small monetary incentive did not significantly improve attendance rates. In Australia up to $85 \%$ of patients visit their GP at least once during a calendar year with most Australians visiting 2-3 times per year $[9,10]$. This means there are potentially many opportunities for GPs to assess cardiovascular risk factors and calculate a cardiovascular risk score. It is possible that the patients we approached had already undertaken a CVRA or at least had their BP, fasting glucose and cholesterol measured. Patients would therefore have little motivation for a repeat assessment. However we know that patients receive only about $60 \%$ of indicated preventive services that are indicated for them [11-14] and a recent study in Australia has shown that there are gaps in recording and managing BP and lipids in Australian general practice [15]. There is also evidence that patients who are at high risk of a cardiovascular event are undertreated [16]. Another explanation may in involve patients' perception of their risk for cardiovascular disease. In a study from the Netherlands many patients were deemed to be at low risk and there was a mismatch between perceived and actual risk with 4 in 5 high risk patients believing incorrectly that were at low risk and 1 in 5 low risk patients believing they were at high risk [17]. This could reduce the number of patients believing that CVRA would be beneficial to them and hence affect attendance rates.

We found that GPs in this study tended to underestimate the CVD risk of their patients. This could be explained if GPs failed to incorporate modifying factors such family history of premature heart disease, impaired glucose tolerance or BMI $>30$ patients' absolute risk. 


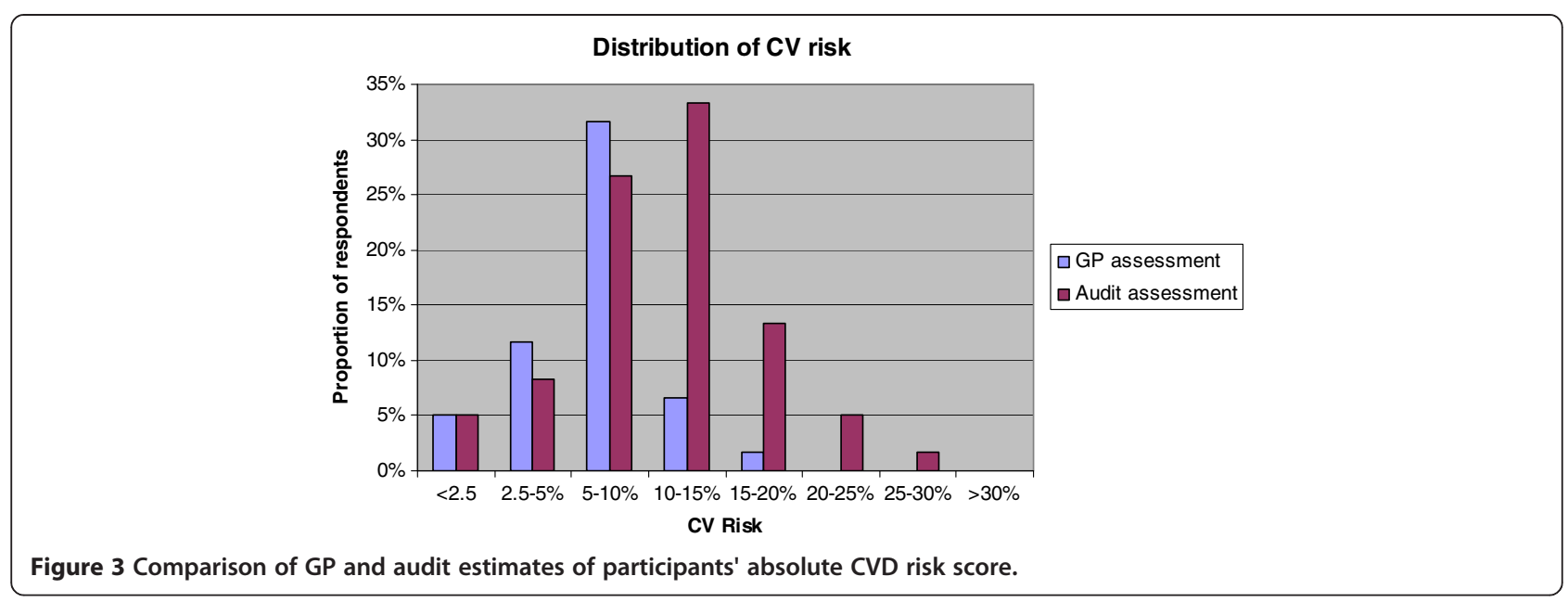

Other studies have demonstrated under and over estimations of CVD risk [18-20]. This may have consequences for the preventive care offered and potentially health outcomes so we should consider better training and/or the use of electronic calculators to improve CVD risk estimation. Our questionnaire also highlighted that inviting patients for CVRA may be worthwhile with almost half indicating that the invitation alone encouraged them to attend and have their 'heart health' assessed.

\section{Limitations}

This study had several limitations. The absolute number of patients responding in the RCT was small although the observed difference in attendance was almost 7\%. Non-attendees may have already had their cardiovascular risk assessed, or may have been concerned that it was a research study. Response rates to a letter(s) or telephone reminder for preventive health care in general practice can vary considerably; for women who had not had a cervical smear in the past 3 years only $10.7 \%$ responded to a letter over 6 months vs $6.3 \%$ in the control [9] whereas for a group of preventive activities the general procedure completion rate was $42 \%$ in a study

Table 3 Participants rating of their own CVD risk in the next 5 years

\begin{tabular}{lllll}
\hline \multicolumn{4}{l}{ Intervention\% } & Control\% \\
\hline $\begin{array}{l}\text { \% } \mathbf{5} \text { year CVD risk on visual analogue } \\
\text { scale }\end{array}$ & 7 & $27 \%$ & 5 & $18 \%$ \\
\hline $0-24 \%$ & 10 & $38 \%$ & 17 & $61 \%$ \\
\hline $25-49 \%$ & 8 & $31 \%$ & 5 & $18 \%$ \\
\hline $50-74 \%$ & 1 & $4 \%$ & 1 & $4 \%$ \\
\hline $75-100 \%$ & 26 & $100 \% 28$ & $100 \%$ \\
\hline Total & & &
\end{tabular}

The $X^{2}$ test for trend (df3) was $2.77 \mathrm{p}=0.428$. from Ottawa. [10] Resource limitations limited our RCT to two practices and prevented sending reminders which have been shown to increase response rates [21]. Invitation letters have been shown to be better than opportunistic health checks in general practice [22] although a Canadian study demonstrated that a telephone invitation to a health awareness program may be better than a letter, but the response to a letter at $44 \%$ was still substantial [23]. The findings from our questionnaire confirm these previous studies.

Finally we could not conduct a double blinded trial because of the obvious difficulties, participants had to be informed about the study and those in incentive arm had to know they would receive a payment if they attended. We did not inform GPs which participants were in the incentive arm, but we could not stop patients discussing this with their doctor. Attendance slips were collected by research staff who did not know which group a patient was in and initial analysis was blinded.

\section{Conclusions}

We believe that this is the first study of its kind in Australia and provides evidence that the use of a small monetary incentive to promote a preventive activity does not work. It is likely that incentives for medication adherence for diagnosed conditions where the benefits of treatment may be more immediately evident and the debate about payments more topical $[24,25]$ - differ from the offer of an incentive for preventive care, where individual benefits are less clear but gains for the community potentially large. Despite this negative result further research with patients is required to determine what, if any, incentives may encourage attendance for preventive activities in primary care. 


\section{Competing interests}

The authors have no competing interests

\section{Authors' contributions}

NS conceived the idea for the study. NS, JA, SW and PR devised the methods and study protocol. JA, SW, OF implemented the study with oversight from NS. NS, JA, SW and PR helped analyse and interpret the results. All authors contributed to the writing and revision of the paper. All authors' read and approved the final manuscript.

\section{Funding}

This project was supported by a Royal Australian College of General

Practitioners Cardiovascular Research Grant.

\section{Acknowledgements}

We would like to acknowledge Dr Nancy Briggs who provided statistical advice to this project and analysed the results. We would also like to thank the Doctors and patients of Hampstead Medical Clinic and Seaton Medical Centre for their participation in this project.

\section{Author details}

'Discipline of General Practice, The University of Adelaide, Adelaide, SA 5005, Australia. ${ }^{2}$ Hills Medical Service, Aldgate 5054, Australia. ${ }^{3}$ Discipline of Public Health, The University of Adelaide, Adelaide, SA 5005, Australia.

Received: 23 December 2011 Accepted: 18 May 2012 Published: 8 June 2012

\section{References}

1. Ebrahim S, Smith G: Multiple risk factor interventions for primary prevention of coronary heart disease. The Cochrane Library 2001, 4:1-27.

2. McAlister FA, Lawson FME, Teo KK, Armstrong PW: Randomised trials of secondary prevention programmes in coronary heart disease: systematic review. BMJ 2001, 323(7319):957-962.

3. Stone EG, Morton SC, Hulscher ME, Maglione MA, Roth EA, Grimshaw JM, et al: Interventions That Increase Use of Adult Immunization and Cancer Screening Services: A Meta-Analysis. Ann Intern Med 2002, 136(9):641-651.

4. Cahill K, Perera R: Competitions and incentives for smoking cessation. Cochrane Database Syst Rev 2008(3). doi:10.1002/14651858.CD004307.pub3. Art. No CD004307.

5. Christensen B: Payment and attendance at general practice preventive health examinations. Fam Med 1995, 27(8):531-534.

6. New Zealand Heart Foundation: Risk Factor Tables; 2008 http://www.nhf.org.nz/index.asp?pagelD=2145828665.

7. Tonkin AM, Lim SS, Schirmer H: Cardiovascular risk factors: when should we treat? MJA 2003, 178:101-102.

8. Jackson R, Lawes CMM, Bennett DA, Milne RJ, Rodgers A: Treatment with drugs to lower blood pressure and blood cholesterol based on an individual's absolute cardiovascular risk. Lancet 2005, 365(9457):434-441.

9. Australian Bureau of Statistics: 0.55 .001 - Health Services: Patient Experiences in Australia.: ; 2009. Available at http://www.abs.gov.au/ausstats/abs@.nsf/ Lookup/4839.0.55.001Chapter12009.

10. Australian Government Department of Health and Ageing: General Practice in Australia. Canberra: Doha; 2005

11. Frank O, Litt J, Beilby J: Opportunistic electronic reminders. Improving performance of preventive care in general practice. Aust Fam Physician 2004, 33:1-2.

12. Australian Institute of Health and Welfare: Cervical screening in Australia 2006-2007. Cancer series no. 47. Cat. no. CAN 43. Canberra: AlHW; 2009.

13. McGlynn EA, et al: The quality of health care delivered to adults in the United States. N Engl J Med 2003, 348(26):2635-2645.

14. Pham $\mathrm{H}$, et al: Delivery of preventive services to older adults by primary care physicians. JAMA 2005, 294(4):473-481.

15. Webster RJ, Heeley EL, Peiris DP, Bayram C, Cass A, Patel AA: Gaps in cardiovascular disease risk management in Australian general practice. MJA 2009, 191:324-329.

16. Heeley EL, Peris DP, Patel A, Cass A, Weekes A, Morgan C, et al: Cardiovascular risk perception and evidence-practice gaps in Australian general practice (theAusHEART study). MJA 2010, 192:254-259.

17. Van der Weijden T, van Steenkiste B, HEJH S, Timmermans DRM, Grol R: Primary Prevention of Cardiovascular Disease in General Practice:
Mismatch between Cardiovascular Risk and Patients Risk Perception. Med Decis Making 2007, 27:724-761.

18. Peters TJ, Montogomery AA, Fahey T: How accurately do primary health care professionals use cardiovascular risk tabels in the management of hypertension? Br J Gen Pract 1999, 49:978-988.

19. McManus RJ, Mant J, Meulendijks CFM, et al: Comparison of estimates and calculation for risk of coronary heart disease by doctors and nurses using different calculation tools in general practice: cross sectional study. BMJ 2002, 324:459-464.

20. Gupta R, Stocks N, Broadbent J: Cardiovascular risk assessment in Australian General Practice. Aust Fam Physician 2009, 5:364-368.

21. Eaker S, Adami H-O, Granath F, Wilander E, Sparen P: A Large PopulationBased Randomized Controlled Trial to Increase Attendance at Screening for Cervical Cancer. Cancer Epidemiol Biomarkers Prev 2004, 13(3):346-354.

22. Norman P: Predicting the uptake of health checks in general practice: invitation methods and patients health beliefs. Soc Sci Med 1993, 37 (1):53-59.

23. Karwalajtys T, Kaczorowski J, Chambers L, Levitt C, Dolovich L, McDonough $B$, et al: A randomized trial of mail vs. telephone invitation to a community-based cardiovascular health awareness program for older family practice patients [ISRCTN61739603]. BMC Fam Pract 2005, 6(1):35.

24. Shaw J: Is it acceptable for people to be paid to adhere to medication? No. BMJ 2007, 335(7613):233.

25. Burns T: Is it acceptable for people to be paid to adhere to medication? Yes. BMJ 2007, 335(7613):232

\section{doi:10.1186/1471-2296-13-54}

Cite this article as: Stocks et al:: Improving attendance for cardiovascular risk assessment in Australian general practice: an RCT of a monetary incentive for patients. BMC Family Practice 2012 13:54.

\section{Submit your next manuscript to BioMed Central and take full advantage of:}

- Convenient online submission

- Thorough peer review

- No space constraints or color figure charges

- Immediate publication on acceptance

- Inclusion in PubMed, CAS, Scopus and Google Scholar

- Research which is freely available for redistribution 